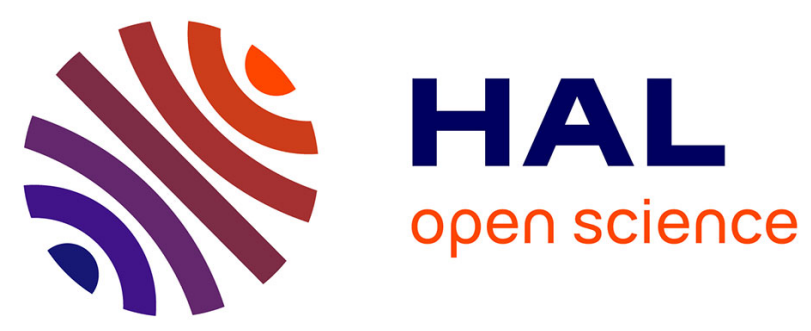

\title{
A ligament laxity telemetry system architecture for a knee replacement prosthesis
}

\author{
Cyril Lahuec, Matthieu Arzel, Manuel Gouriou, François Gallée
}

\section{To cite this version:}

Cyril Lahuec, Matthieu Arzel, Manuel Gouriou, François Gallée. A ligament laxity telemetry system architecture for a knee replacement prosthesis. 2nd International Symposium on Applied Sciences in Biomedical and Communication Technologies, Nov 2009, Bratislava, Slovakia. pp.1 - 6. hal-01801574

\section{HAL Id: hal-01801574 https://hal.science/hal-01801574}

Submitted on 28 May 2018

HAL is a multi-disciplinary open access archive for the deposit and dissemination of scientific research documents, whether they are published or not. The documents may come from teaching and research institutions in France or abroad, or from public or private research centers.
L'archive ouverte pluridisciplinaire HAL, est destinée au dépôt et à la diffusion de documents scientifiques de niveau recherche, publiés ou non, émanant des établissements d'enseignement et de recherche français ou étrangers, des laboratoires publics ou privés. 


\title{
A ligament laxity telemetry system architecture for a knee replacement prosthesis
}

\author{
Cyril Lahuec, Matthieu Arzel, Manuel Gouriou, François Gallée \\ TELECOM Bretagne \\ Brest, Brittany, France \\ Email: cyril.lahuec, matthieu.arzel, manuel.gouriou, francois.gallee@telecom.bretagne.eu
}

\begin{abstract}
In this paper the feasibility of a novel instrumented orthopaedic implant for total knee replacement is studied. The system aims at providing an indication on the balance of the lateral ligaments. The measures will be used to estimate the wear out of the polyethylene part of the prosthesis and will also facilitate the patient rehab. The data will be transmitted outside the body through an RF link. Unlike other instrumented implants, the system will be self-powered. Piezo ceramics will be used both as pressure sensors and as power generators. This implies that the measurement system must be ultra low power as the available power is less than $1.8 \mathrm{~mW}$. First simulation results are given for a $0.35 \mu \mathrm{m}$ CMOS process.
\end{abstract}

\section{INTRODUCTION}

Total knee arthroplasty (TKA) is used to replace a damaged knee joint with metallic parts fixed or impacted over the bones and separated by a polyethylene part. The causes of damage are wide but the most common is osteoarthritis. During surgery any defect must be corrected to insure a proper functioning of the implant. In particular the collateral ligaments must be balanced in order to insure proper knee movements and stability. Imbalanced ligaments cause an early wear of the polyethylene part and could, in the end, cause the loosening of the tibial tray. Unfortunately, the skill of the surgeon is not enough to insure a perfect balance. In order to avoid injury due to a loosening of the implant, it seems interesting to be able to predict the lifespan of the prosthesis by measuring the forces applied onto the tibial tray. In-vivo force measurements have been shown to be possible [1][2]. These systems use strain gauges. The systems only measure and output the magnitude of the axial force applied. They do not provide any clue about the polyethylene wear. Most of the published smart orthopaedic implants use power hungry offthe-shelf components; they are hence powered by cumbersome inductive coil. The dimension of the coils used in such systems depends on the application, the losses are also important due to the presence of large metallic parts [2]. This paper presents a possible architecture of a self-powered telemetry system performing in-vivo interpretation of the measured forces to estimate the balance of the lateral ligaments. Then, from polyethylene wear data from implant manufacturers it will be possible to estimate the life expectancy of the prosthesis. Although some of the blocks in the architecture have not yet been fully evaluated, it is still possible to estimate the feasibility of such a system.

The paper is organized as follows. Part II describes the sensor

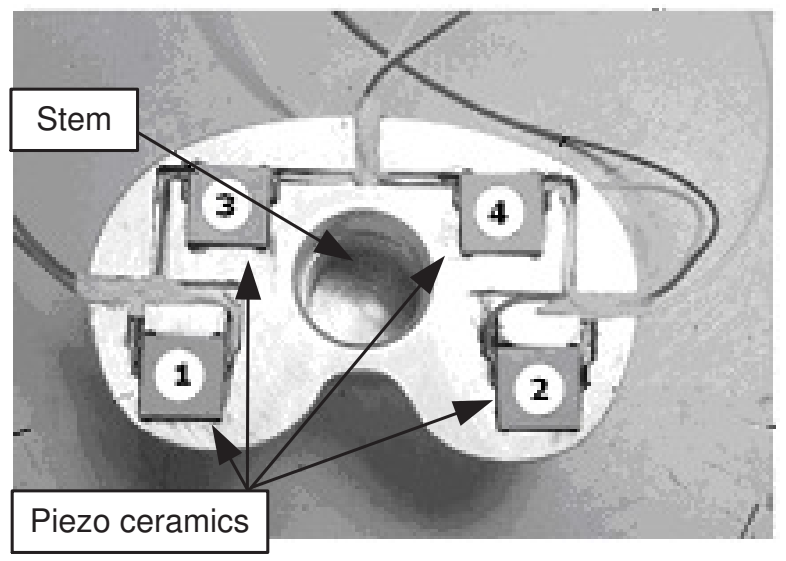

Fig. 1. Tibial tray prototype fitted with four piezo ceramics SCMA P09 of dimensions $10 \mathrm{~mm} \times 10 \mathrm{~mm} \times 4 \mathrm{~mm}$. Piezo ceramics 1 and 3 internal compartment, piezo ceramics 2 and 4 external compartment.

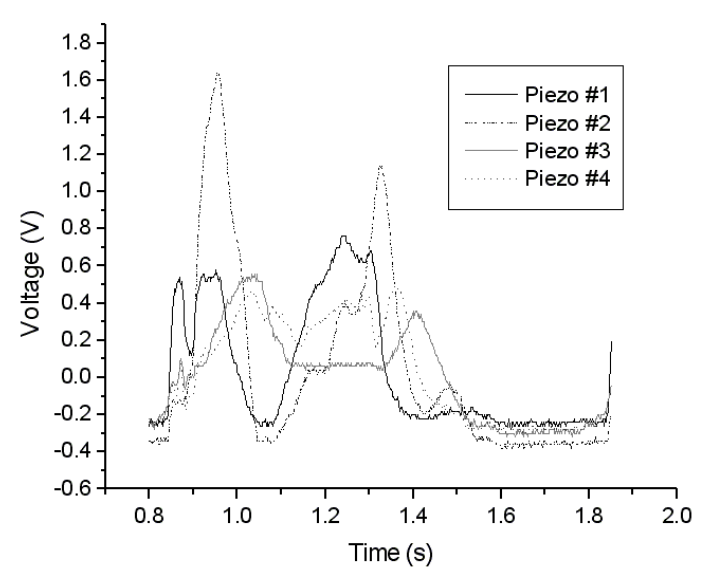

Fig. 2. Measured voltages representing forces over one walking cycle on a $10 \mathrm{k} \Omega$ resistor, balanced prosthesis, person weight $88 \mathrm{~kg}$.

and the source of energy. Part III deals with the measuring method. In part IV a description of the proposed ASIC is done. Part V shows preliminary simulation results of the computing core. Part VI deals with the estimated power consumption. Finally, part VII concludes the paper. 


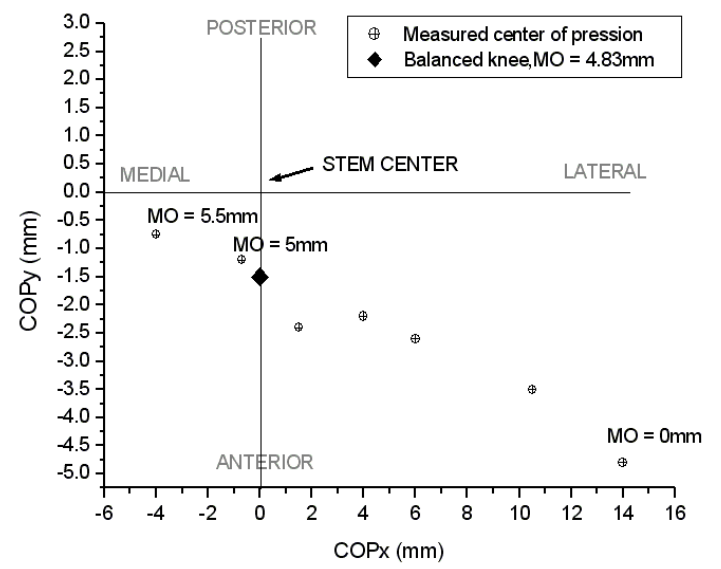

Fig. 3. Displacement of the center of pressure (COP) for various medial offsets (MO). The point $(0,0)$ represents the center of the stem. The position of the COP for the ideal $\mathrm{MO}=4.83 \mathrm{~mm}$ is represented with a diamond.

\section{KNEE PROSTHETIC PROTOTYPE}

\section{A. Piezo ceramics}

The voltage across a piezo ceramic when mechanically stressed is proportional to the force applied. Hence, they can be used both as force sensor and as energy generator as proposed in [3]. The huge force applied onto the tibia, up to four times the person weight [4], can be used to harvest enough energy to power the telemetry system. In [5], three piezo ceramics were used to yield $2 \mathrm{~mW}$, enough to power a microcontroller. Platt was not concerned with measuring forces but with generating power, so the piezos were placed near the center of the tray. To obtain the axial force distribution over the tibial tray in both the medio-lateral (M-L) and the antero-posterior (A-P) directions, the tray is divided in four areas in which a piezo ceramic is placed, Fig. 1. One pair of piezo ceramics is located in the internal compartment (piezo ceramics 1 and 3) and the other in the external compartment (piezos 2 and 4). The prime concern of this work was to obtain a suitable metric to estimate the polyethylene wear out. Moreover, it was not wished to drastically modified the design of the tray as Platt did. Hence the choice of $10 \mathrm{~mm} \times 10 \mathrm{~mm} \times 4 \mathrm{~mm}$ SCMA P09 so that they can be embedded into a normal $5 \mathrm{~mm}$-thick tray although they would generate less than $2 \mathrm{~mW}$.

\section{B. Measurements}

The prototype was fitted onto a MTS 858 MINI BIONIX I knee simulator both to estimate the amount energy that could be harvested and to find a metric suitable to estimate the ligaments laxity from the force measurements. Figure 2 shows the four output voltages representing the forces applied onto the piezo ceramics when the knee is balanced and for a $88 \mathrm{~kg}$ person. Based on these signals, the average power generated over a walking cycle has a maximum value of $0.36 \mathrm{~mW}$ on a $35 \mathrm{k} \Omega$ resistor. With $7 \mathrm{~mm} \times 7 \mathrm{~mm} \times 8 \mathrm{~mm}$ piezos, the proposed
TABLE I

PIEZO CERAMICS CENTER WITH RESPECT TO THE CENTER OF THE STEM (IN MM)

\begin{tabular}{|l||c|c|}
\hline Piezo ceramic $i$ & $X_{\text {piez }}$ & $Y_{\text {piez }}$ \\
\hline \hline 1 & -22.25 & -13.25 \\
\hline 2 & 22.25 & -13.25 \\
\hline 3 & -17.25 & 8.75 \\
\hline 4 & 17.25 & 8.75 \\
\hline
\end{tabular}

method yields $1.8 \mathrm{~mW}$ at the expense of thicker tray, $9 \mathrm{~mm}$ instead of $5 \mathrm{~mm}$.

\section{USEFUL METRIC}

\section{A. Medial offset and center of pressure}

In a balanced knee, the internal condyle of the femur applies more efforts on the tibial tray than the external condyle. This corresponds to a medial offset (MO) in the application of the axial force of $0.07 \times W$ from the tibia axis [4], $W$ the width of the tray. In the present case $W=69 \mathrm{~mm}$, which corresponds to a $\mathrm{MO}$ of $4.83 \mathrm{~mm}$. What the surgeon is interested in is to estimate the life expectancy of the implant, is the actual shift from the ideal MO due to inaccurate implant fitting causing ligament imbalance. Any ligament imbalance modifies the MO and hence the axial force distribution on the tibial tray. The four data provided by the piezo ceramics can be combined into a single one by calculating the barycenter of the forces measured at each piezo ceramic. The barycenter, or center of pressure (COP), indicates on which part of the tray the axial force is mostly applied. The COP is thus the sum of the voltages weighted by the physical center of each piezo taking the center of the stem as origin.

\section{B. Computing the center of pressure}

The coordinates of the barycenter are given by the mediolateral (M-L) $C O P_{x}$ and the antero-posterior (A-P) $C O P_{y}$ displacements described by (1) and (2), respectively.

$$
\begin{aligned}
C O P_{x} & =\frac{\overline{V_{1}} X_{\text {pie }_{1}}+\overline{V_{2}} X_{\text {piez }_{2}}+\overline{V_{3}} X_{\text {piez }_{3}}+\overline{V_{4}} X_{\text {piez }_{4}}}{\sum_{i=1}^{4} \overline{V_{i}}} \\
C O P_{y} & =\frac{\overline{V_{1}} Y_{\text {piez }_{1}}+\overline{V_{2}} Y_{\text {pie } z_{2}}+\overline{V_{3}} Y_{\text {piez }_{3}}+\overline{V_{4}} Y_{\text {pie }_{4}}}{\sum_{i=1}^{4} \overline{V_{i}}}
\end{aligned}
$$

where $\overline{V_{i}}$ is the average voltage over one walking cycle recorded at piezo number $i, X_{\text {piez }}$ and $Y_{\text {piez }}$ are the coordinates of the center of piezo $i$ which are given in Table I.

When the knee is perfectly balanced, i.e. for an offset of $4.83 \mathrm{~mm}$ the axial force is evenly distributed on the tray, the coordinates of the center of pressure are $C O P_{x}^{\text {balanced }}=0 \mathrm{~mm}$ and $C O P_{y}^{\text {balanced }}=-2.25 \mathrm{~mm}$. Then the offset is changed from 0 to $5.5 \mathrm{~mm}$ to shift the axial force application axis to simulate the ligament imbalance and the average COP is computed. This is represented in Fig. 3. The case for which the MO is $0 \mathrm{~mm}$ never happens in practice but is given for reference. 


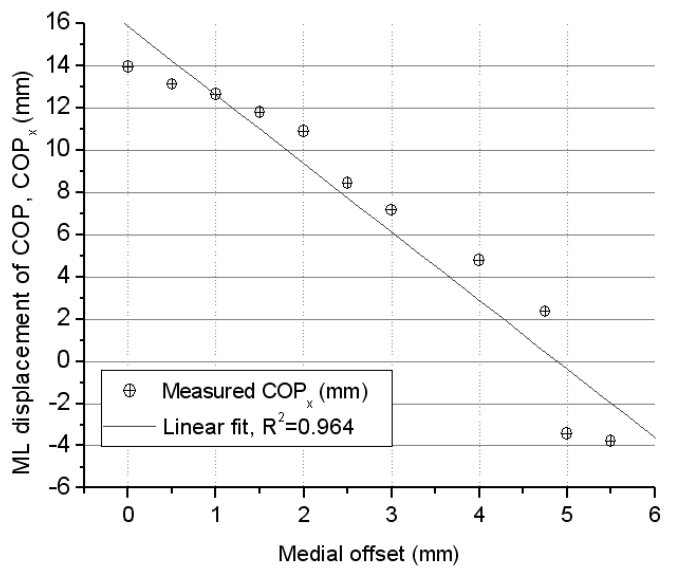

Fig. 4. Average M-L displacement of the center of pressure (COP) for various medial offsets (MO).

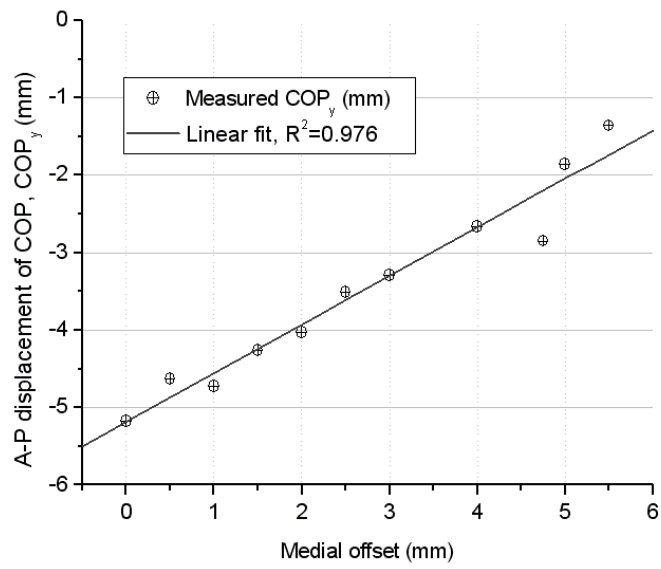

Fig. 5. Average A-P displacement of the center of pressure (COP) for various medial offsets (MO).

\section{Simplification}

Although the required calculations to perform are relatively simple, for the sake of power it is worth trying to simplify them. The data collected allow for medio-lateral (M-L) and antero-posterior (A-P) displacement of the COP. These data are plotted in Fig. 4 and Fig. 5, for the average M-L and A$\mathrm{P}$ displacements respectively. From these figures, the relation between the COP and the MO is almost linear. Hence, knowing the COP, it is possible to obtain the corresponding MO by linear fit. Only the M-L displacement could be used since it has a larger dynamic than the A-P displacement. It is thus easier to discriminate the medial offsets from one another using a simple linear fit. Therefore, the processing unit will only implement (1).

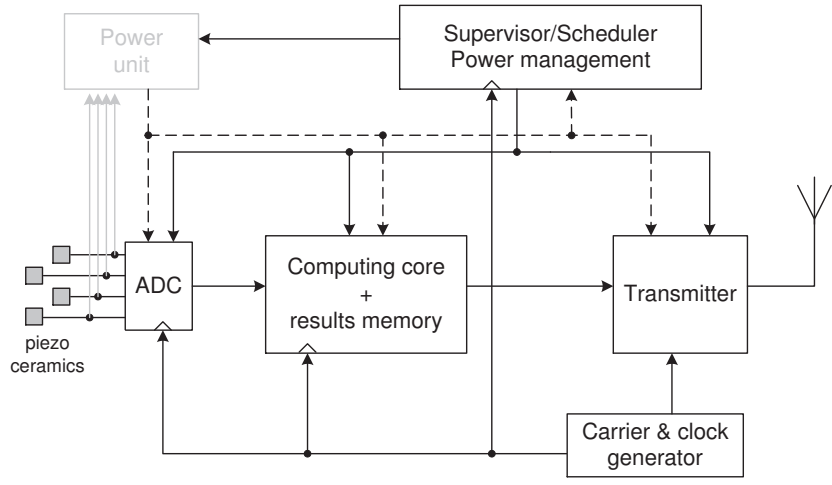

Fig. 6. Block diagram of the ligament balance measuring system.

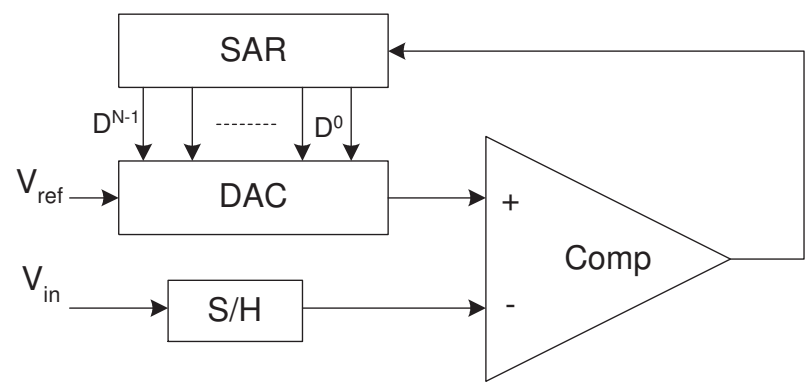

Fig. 7. SAR ADC block diagram.

\section{PROCESSING UNIT}

Considering the low average power available it is necessary to design a dedicated circuit. It should include the required analog interface functions, i.e. analog-to-digital converter and a transceiver as well as the digital data processing core. Since it is expected that most of the power will be used by the transmission link, a careful design of the processing unit is required to insure that the data conversion and the core consume the least power possible. The general architecture is shown in Fig. 6. At this stage of the project, the system will only output the value of $C O P_{x}$, the calculation of the corresponding MO is done on the medical staff's computer.

\section{A. Supervisor core}

The supervisor core is basically a 4-state machine scheduling the rest of the circuit. The supervisor block generates all the control signals for each part and, if need be, switches on or off the different parts of the measuring system. Although static leakage current is of no concern for the technology used [6], power gating could be used to redirect all the available power to one part of the system at a specific time, to the transceiver when emitting the data for instance. This might not be necessary if the system is designed for ultra low power consumption as power gating introduces a lot of noise in the system and might require a specific wake-up schedule [7]. This block must be on all the time. 

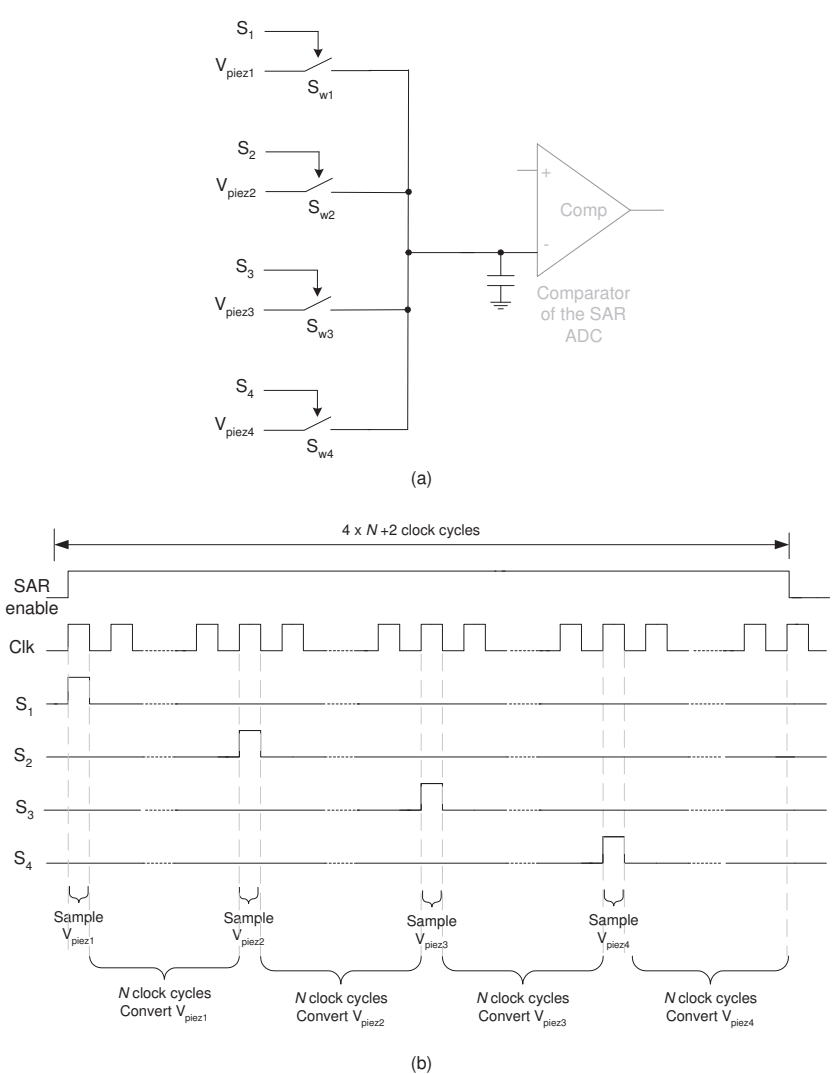

Fig. 8. Simplified Sample-and-Hold block with corresponding timing diagram.

\section{B. Analog to digital converter}

The chosen architecture for the analog-to-digital converter is a successive approximation register (SAR) as it is the most efficient in terms of power consumption, few $\mu \mathrm{W}$ for rates up to $500 \mathrm{kS} / \mathrm{s}$, [8]-[11]. The block diagram of the ADC is given in Fig. 7, its functioning is briefly explained. The value $V_{i n}$ to convert is sampled and held. The MSB of the $N$ bit SAR, $D^{N-1}$, is initialized at 1 and all the other bits at 0 . This digital word corresponds to $V_{\text {ref }} / 2$, which is fed to the non-inverting input of the comparator through a digitalto-analog converter and compared to the sampled voltage. If $V_{\text {in }}<V_{\text {ref }} / 2$, then $D^{N-1}$ is reset, the next bit, $D^{N-2}$, is set to 1 and the remaining bits stay at 0 . If $V_{\text {in }}>V_{\text {ref }} / 2$, then $D^{N-1}$ remains at $1, D^{N-2}$ is set to 1 and the remaining bits stay at 0 . The search continues until each bit in the SAR has been tested. Hence, it takes $N$ clock cycles to perform a conversion. The DAC is implemented as a binary-weighted capacitor array that uses a unit capacitor $\mathrm{C}$. To reduce on chip area and power consumption, a single ADC is used so that the four signals are sampled and converted in turns. The $\mathrm{S} / \mathrm{H}$ block and its timing diagram are given in Fig. 8. Half a cycle is used to sample each piezo voltage, the next $N$ clock cycles are used to convert the value into a digital word. An overall of $4 \times \mathrm{N}+2$ clock cycles is thus necessary to encode the four piezo voltages, a period of time during which the SAR ADC is enabled. Ideally, the four piezo signals should be sampled at the same time. Reducing the conversion time of the ADC for the four samples to a small fraction, one tenth for instance, of the sampling period helps approaching the ideal case. This helps reducing the energy consumption.

\section{Computing core}

The digital core computes the barycenter as described in section from which it is then possible to know the offset from the ideal position. The block diagram of the digital is shown in Fig. 9. The digital core is divided into three parts. The first part computes the average voltage over one walking cycle for each piezo using an adder-accumulator. The average value is obtained by dividing the cumulated sum by the number of samples $N_{S}$. To simplify the design, $N_{S}$ is a power of 2 . Only shifts are then necessary to obtain the average. This part of the circuit needs to run after each conversion, so four times during the $4 \times N+2$ clock cycles and this $N_{S}$ times per walking cycle. The first part of the digital core runs thus at $4 \times N_{S}$ in the example taken. The average voltages $\overline{V_{i}}$ are then transfered to the second and third blocks to compute in parallel the numerator and the denominator, respectively, of (1). The denominator of (1) is simply obtained through a tabulated inverse. The partial results are then multiplied to produce the average $\mathrm{M}-\mathrm{L}$ displacement $C O P_{x}$, which value is stored in a memory. This part only runs once per walking cycle. The number of bits required for each step, i.e. $N, n, m$, $t$ and $d$ in Fig. 9 is optimised through behavioral simulations in order to reduce the power consumption.

\section{Transmitter}

This block reads out the result memory from the computing core module and transmits outside the patient's body. The transmitter must comply with standard ETSI 301-839 for Medical Implant Communication Systems. The allowed frequency band is $402 \mathrm{MHz}-405 \mathrm{MHz}$ with a maximum effective radiated power to $25 \mu \mathrm{W}$ [12]. Some architectures for such block have been proposed for this standard, but they are not suitable considering the available power budget [13]. A much simpler architecture is thus chosen, Fig. 10. The design of this block requires to take into account several parameters. First, the various human tissues with high permittivity (bones, muscles, fat, skin) do not help with RF transmission. Placed in a similar environment, planar inverted-F antennas (PIFA) have been shown to be smaller and have a higher radiation efficiency than other types of antennas [14]. Second, the antenna should be designed taking the metallic nature of the implant. Third, a power efficient modulation scheme, such as Binary Phase Shift Keying should be used. All these will relax the PA design. Finally, the PA and antenna will also constraint the sensitivity of the receiver frond-end. It is not planned yet to receive data, such as configuration bits, from the outside. If a transceiver is required, a simple receiver architecture, such as heterodyne architecture, should be implemented, as shown in grey in Fig. 10 . 


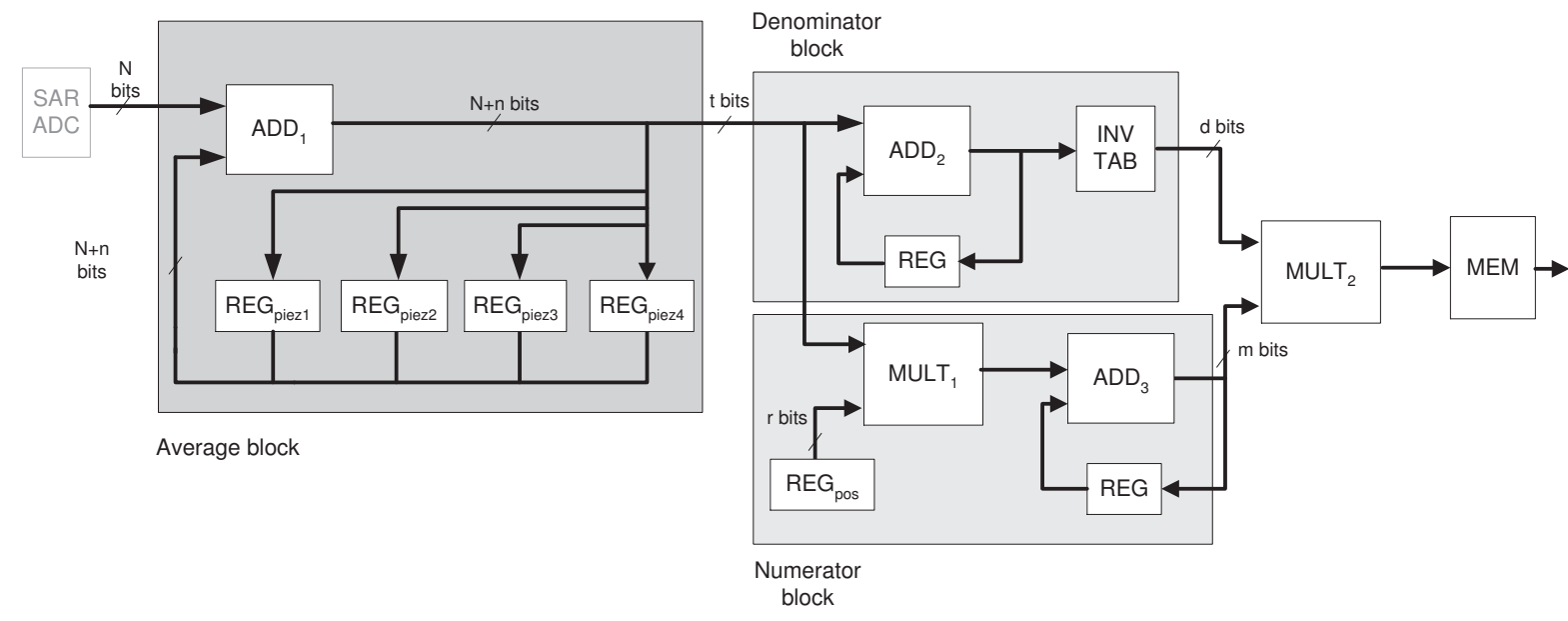

Fig. 9. Digital core computing the average M-L displacement $C O P_{x}$.

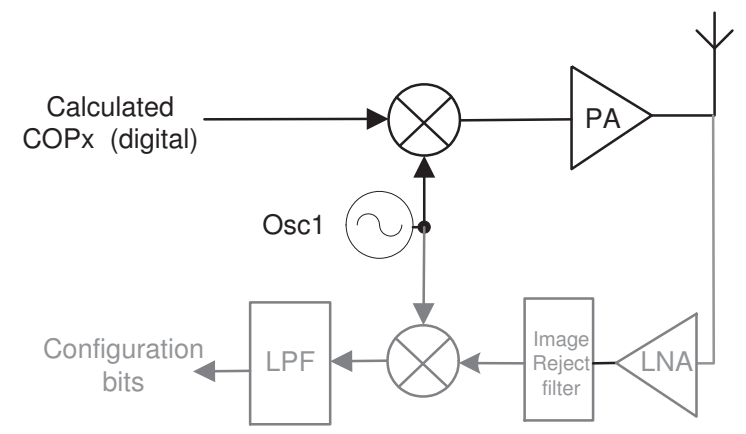

Fig. 10. Simple transmitter, using BPSK modulation scheme. In grey, possible receiver architecture.

TABLE II

DATA FORMAT

\begin{tabular}{|l||c|}
\hline Name & Number of bits \\
\hline$N$ & 6 \\
\hline$d$ & 9 \\
\hline$n$ & 9 \\
\hline$m$ & 10 \\
\hline$r$ & 4 \\
\hline$t$ & 6 \\
\hline
\end{tabular}

\section{E. Carrier and clock generator}

Besides the supervisor core the carrier and clock generator needs also to be on all the time to avoid transient in the oscillating frequency after switching on the oscillator. This block must thus be well thought to consume the least power. Two signals must be generated, one for the transmitter at $402 \mathrm{MHz}$ and the other at a much lower frequency, in the $\mathrm{kHz}$ range to clock the computing core. Due to the large frequency ratio it might be interesting for power reasons to implement a second low power low frequency oscillator.

\section{Simulation Results}

At this stage of the work only the ADC and the computing core have been simulated. The accuracy of the system depends on the number of bits in the ADC and on the implementation of the computing core. The calculations done to produce Fig. 4 are used as a reference, they correspond to a floating point representation. The computing core will implement a fixed point representation which implies trade-off between accuracy and complexity. A behavioral $\mathrm{C}$ model is used for that purpose. Referring back to Fig. 9, the number of samples is chosen to be $N_{S}=128$. The number of conversion bits $N$ directly affects the rest of the circuit. Simulations shows that a 6-bit ADC is enough, hence the total number of bits required at the output of $A D D_{1}$ is 16 , considering that 128 values are added per piezo. However, the MSB can be removed as the average value of the samples will never reach half the maximal value, i.e. $n=9$. This leads to the removal of a half-adder in the $A D D_{1}$ block and a flip-flop in registers $R E G_{\text {piez2 }}$ to $R E G_{\text {piez } 4 \text {. }}$. Next, the number of bits going from the Average block to the Denominator and Numerator blocks is reduced by noticing that the average force value per piezo changes significantly only when in the vicinity of the balanced point. Hence, only the six MSB $(t=6)$ can be kept without affecting the result. Following the same procedure, the data format through out the computing core are obtained and are listed in Table II. The computed medial offset using this system is compared to the one computed using the floating point representation versus the actual medial offset in Fig. 11. Despite simplifying the system a lot, there is still a good agreement between the two.

\section{POWER CONSUMPTION}

A $0.35-\mu \mathrm{m}$ CMOS technology from AMS is chosen to design the ASIC with a supply voltage of $1.3 \mathrm{~V}$. From the design kit it is possible to accurately estimate the power consumption of the digital parts to about $0.003 \mathrm{~mW}$. From the discussion on the $\mathrm{ADC}$, the power consumption of the $\mathrm{ADC}$ is at most $1 \mu \mathrm{W}$ when working. The ADC clock period is $33 \mathrm{kHz}$, choosing $N_{S}=128$. Hence, the worst case power consumption of the digital core is estimated to $0.003 \mathrm{~mW}$. Building the $402 \mathrm{MHz}$ 


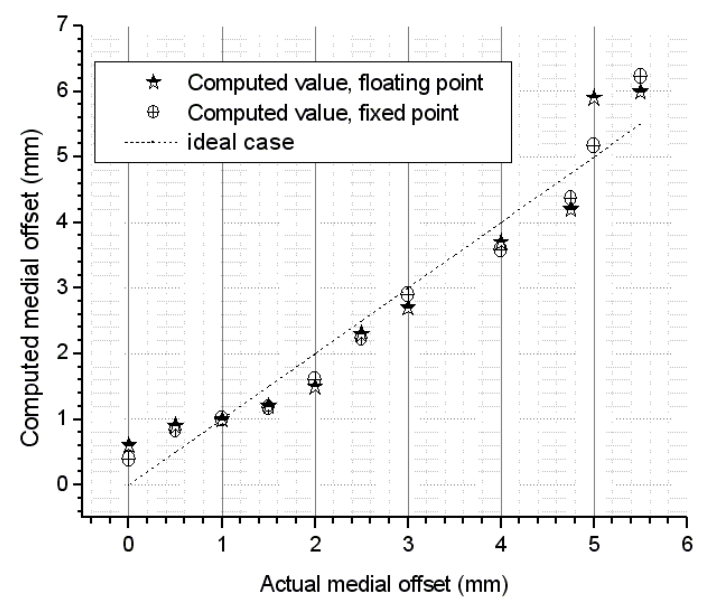

Fig. 11. Comparison between the computed medial offsets using floating point (corresponding to Fig. 4) and fixed point logic versus the actual medial offset.

TABLE III

POWER LEFT FOR TRANSMITTER TO ESTIMATE RECEIVER SENSITIVITY.

\begin{tabular}{|l|c|}
\hline Available power $(\mathbf{m W})$ & +1.81 \\
\hline Consumed power (mW) & $-1 \mathrm{E}-3$ \\
SAR & $-3 \mathrm{E}-3$ \\
Digital core (computing + supervisor) & -0.1 \\
Oscillator 1 (402MHz ring) & -0.1 \\
Oscillator 2 (33kHz harmo.) & +1.6 \\
\hline Power left for transmitter (mW) & \\
\hline
\end{tabular}

carrier and the $33 \mathrm{kHz}$ clock from a single $402 \mathrm{MHz}$ oscillator, which should not consume more than $100 \mu \mathrm{W}$ [15], implies a large and fast frequency divider. This divider would consume more than a second harmonic oscillator consuming about $100 \mu \mathrm{W}$ [16]. Therefore, two oscillators will be used. This is not a problem since there is no synchronisation requirement between the acquisition/computing part and the transmitting part. Table III gives the estimated power consumption of each block and what is left for the transmitter if everything on at the same time (which will not be the case), at most $1.6 \mathrm{~mW}$. Considering a seven percent power amplifier efficiency, an isotropic antenna with a one percent radiation efficiency and a $30 \mathrm{~cm}$ free-space link as in [14] then the exterior reading device sensitivity of the receiver, using a simple dipole antenna, should be below $-41 \mathrm{dBm}$ to achieve a reliable link. Most RF receivers in the 300 to $900 \mathrm{MHz}$ range achieve input sensitivity below $-90 \mathrm{dBm}$ such as the AMIS-53000 transceiver chip for medical application from ON Semiconductor [17]. Hence the received signal level is well above what can be achieved by commercial receivers.

\section{CONCLUSION}

This paper has presented a possible ASIC architecture for measuring the line of application of the axial force medial offset from force measurements on the tibial tray. As the simulation of the computing shows, despite many simplifications, it is possible to match the measurements. As expected the most difficult part to define is the transceiver. It cannot be designed without designing also the exterior receiving device, a system level approach is necessary. Moreover, the implanted antenna has to be designed taking the nearby metallic tibial tray into account. However, the proposed telemetry system can be done. The $1.6 \mathrm{~mW}$ left for the transmitter allows for implementing the linear fit on-chip and output the MO directly. Also, a full transceiver could be implemented.

\section{REFERENCES}

[1] S. Taylor, J. Gorjon, and P. Walker, "An instrumented prosthesis for knee joint force measurement in vivo," in Innovative Pressure, Force and Flow Measurements (Ref. No. 1999/089), IEE Colloquium on, 1999, pp. 6/1-6/4.

[2] F. Graichen, R. Arnold, A. Rohlmann, and G. Bergmann, "Implantable 9channel telemetry system for in vivo load measurements with orthopedic implants," Biomedical Engineering, IEEE Transactions on, vol. 54, no. 2, pp. 253-261, Feb. 2007.

[3] M. Gouriou, S. Almouahed, C. Hamitouche, C. Roux, and E. Stindel, "Predictive autonomous orthopaedic device," in $9^{\text {th }}$ Workshop on Computer Assisted Orthopeadic Surgery, Boston, USA, June 2009, pp. -.

[4] ISO14243-1:2002(E), "Implants for surgery-wear of total knee joint prostheses-part 1: Loading and displacement parameters for wear-testing machines with load control and corresponding environmental conditions for test," International Organization for Standardization, Mar. 2002.

[5] S. Platt, S. Farritor, K. Garvin, and H. Haider, "The use of piezoelectric ceramics for electric power generation within orthopedic implants," Mechatronics, IEEE/ASME Transactions on, vol. 10, no. 4, pp. 455461, Aug. 2005.

[6] G. Panic, Z. Stamenkovic, and R. Kraemer, "Power gating in wireless sensor networks," in Wireless Pervasive Computing, 2008. ISWPC 2008. 3rd International Symposium on, May 2008, pp. 499-503.

[7] M.-C. Lee, S.-C. Chang, C.-S. Su, and E. Tsai, "Performance and wake-up schedule optimization of power gating design," in SoC Design Conference, 2008. ISOCC '08. International, vol. 01, Nov. 2008, pp. I-36-I-39.

[8] T. K. H. Roy and T. Teo, "A 0.9v 100nw rail-to-rail sar adc for biomedical applications," in Integrated Circuits, 2007. ISIC '07. International Symposium on, Sept. 2007, pp. 481-484.

[9] K. Abdelhalim, L. MacEachern, and S. Mahmoud, "A nanowatt successive approximation adc with a calibrated capacitor array for biomedical applications," in Circuits and Systems, 2007. MWSCAS 2007. 50th Midwest Symposium on, Aug. 2007, pp. 136-139.

[10] Y.-K. Chang, C.-S. Wang, and C.-K. Wang, "A 8-bit 500-ks/s low power sar adc for bio-medical applications," in Solid-State Circuits Conference, 2007. ASSCC '07. IEEE Asian, Nov. 2007, pp. 228-231.

[11] N. Verma and A. Chandrakasan, "An ultra low energy 12-bit rateresolution scalable sar adc for wireless sensor nodes," Solid-State Circuits, IEEE Journal of, vol. 42, no. 6, pp. 1196-1205, June 2007.

[12] ETSI-EN-301-839-1, "Electromagnetic compatibility and radio spectrum matters (erm);radio equipment in the frequency range $402 \mathrm{mhz}$ to 405 mhz for ultra low power active medical implants and accessories;part 1: Technical characteristics, including electromagnetic compatibility requirements, and test methods." 2002.

[13] P. Bradley, "An ultra low power, high performance medical implant communication system (mics) transceiver for implantable devices," in Biomedical Circuits and Systems Conference, 2006. BioCAS 2006. IEEE, 29 2006-Dec. 1 2006, pp. 158-161.

[14] J. Kim and Y. Rahmat-Samii, "Implanted antennas inside a human body: simulations, designs, and characterizations," Microwave Theory and Techniques, IEEE Transactions on, vol. 52, no. 8, pp. 1934-1943, Aug. 2004.

[15] M. Rashdan, M. Pai, P. Anand, J. Haslett, and B. Maundy, "Ultra low power transceiver for wireless patient vital sign monitoring," in Electrical and Computer Engineering, 2007. CCECE 2007. Canadian Conference on, April 2007, pp. 425-428.

[16] H. Fan, "On-chip real time clock oscillator without quartz cristal," Ph.D. dissertation, Télécom Bretagne, Brest, Brittany, France, Oct. 2009.

[17] ON-Semiconductor, "Amis-530xx frequency agile transceiver etsi test report," www.onsemi.com, May 2008. 